\title{
An Invitro Evaluation of the Accuracy of Two Electronic Apex Locators to Determine Working Length in the Presence of Various Irrigants
}

\author{
Prasad Ashwini B ${ }^{1}$, Srivastava Harshit ${ }^{2}$, Srivastava Arora Aastha ${ }^{2}$, Raisingani \\ Deepak $^{3}$
}

ABSTRACT

BACKGROUND: Cleaning and shaping of root canal system requires various irrigating solutions. The question is whether the presence of irrigation solution alters working length determination using apex locators. The purpose of this study was to compare the accuracy of Root $Z X$ and $i$-Root apex locator for determining working length in the presence of different irrigating solution.

MATERIALS AND METHOD: Eighty extracted single rooted human teeth were used. The teeth were sectioned at Cemento Enamel Junction (CEJ) and actual canal length determined. Then, working length measurements were obtained using Root ZX and i-Root apex locator in the presence of irrigating solutions namely $0.9 \%$ saline, $3 \%$ of sodium hypochlorite (NaOCl), $2 \%$ chlorhexidine digluconate $(\mathrm{CHX})$ and $17 \%$ Ethylene Diamine Tetra Acetic acid (EDTA). The measurements obtained with Root $\mathrm{ZX}$ and $\mathrm{i}$-Root apex locator were compared with actual canal length and subjected to statistical analysis using Post Hoc Test Tukey's Method.

RESULTS: This study revealed that both the tested Electronic apex locators (EAL) were able to measure the canal length in the presence of tested irrigating solutions. The presence of irrigating solutions of saline, $\mathrm{NaOCl}$, chlorhexidine and EDTA in the root canal marginally influenced the accuracy of the Root $\mathrm{ZX}$ or $i-\operatorname{Root}(P<0.36)$, but with no clinical significance.

CONCLUSION: Root ZX and i-Root can be used safely to determine working length in the presence of various irrigants. The content of the root canal did not influence the accuracy while measuring working length using Electronic apex locators (EAL)

KEYWORDS: Chlorhexidine, Root ZX, i-Root Apex locator, sodium hypochlorite, saline

\section{DOI: http://dx.doi.org/10.4314/ejhs.v26i5.7}

\section{INTRODUCTION}

Preparation of canal, especially in the apical segment, without weakening the remaining dentin or perforating the root is essential for proper disinfection. To achieve this, virtually all steps of root canal therapy demand strict working length to ensure that neither the root canal system nor the periodontal ligament is damaged (1).

Hence, the accurate working length determination has a profound effect on the root canal preparation, microbial disinfection and hermetic seal of the root canal system. Correct canal length is also necessary to minimize the extrusion of potentially infected debris into the periapical area. The outcome of treatment of roots with necroticpulps and periapical lesions is significantly influenced by the apical level of the root canal filling (2).

Cluster was the first to develop the idea that root canal length could be estimated by the use of an electrical current. Suzuki discovered that the

\footnotetext{
${ }^{1}$ Associate Professor, Department of Conservative Dentistry and Endodontics, Mahatma Gandhi Dental College, Jaipur, India

${ }^{2}$ Assistant Professor, Departmrnt of Conservative Dentistry and Endodontics, Mahatma Gandhi Dental College \& Hospital, Jaipur, India

${ }^{3}$ Professor \& Head of the Department, Department of Conservative Dentistry and Endodontics, Mahatma Gandhi Dental College, Jaipur, India

Corresponding Author: Ashwini B Prasad, Email: catchashu@rediffmail.com
} 
electrical resistance between the periodontal ligament and the oral mucosa was a constant value of $\sim 6.5 \mathrm{k} . \Omega$. (4)

Sunada introduced the principle of 'biological characteristics theory' to clinical practice. Based on this research, Sunada demonstrated that a constant resistance value of $6.2 \mathrm{k} \Omega$ is established between the mucous membrane and periodontium irrespective of age, sex, tooth and canal curvature of the patient (5).

Problems inherent in using direct current (resistance based, $1^{\text {st }}$ generation apex locators) led to the development of apex locators which used alternating current (Single frequency impedance, $2^{\text {nd }}$ generation apex locators). These second generation apex locators have the major disadvantage that the canal needs to be reasonably free of electrically conductive material like blood for an accurate reading. The presence of tissue and conductive irrigant in the canal leads to measurement error (6).

The third-generation or multiple frequencydependent apex locators use alternate currents. Based on either difference of impedance (Eg Endex) or ratio of impedance (Eg.Root ZX) between electrodes.

The Root ZX (J. Morita Corp. Kyoto, Japan) uses two different frequencies $(8 \mathrm{kHz}$ and 0.4 $\mathrm{kHz}$ ) to simultaneously measure the impedances in the canal. The device determines a quotient value by dividing the $8 \mathrm{kHz}$ impedance value by the 0.4 $\mathrm{kHz}$ impedance value. The minor diameter is located when the quotient equals 0.67. By using two frequencies, the Root ZX can be used in all types of fluids because the quotient $(0.67)$ is always the same (7).

i-Root (S-Denti Co. Ltd Seoul, Korea) apex locator has different frequencies-5 KHz and 500 Hz. The manufacturer claims that its accuracy is good, irrespective of canal contents.

Saline, $\mathrm{NaOCl}$, chlorhexidine digluconate and EDTA are commonly used for irrigation of root canals.This study was conducted to test the accuracy of two frequency based apex locators, namely, Root ZX and i-Root in the presence of these commonly used irrigants. The purpose was to find out whether the presence of irrigants affected the device measurements.

\section{MATERIALS AND METHOD}

Before this invitro study was conducted, ethical approval was acquired from the Institutional Research Review Board of Jaipur Dental College. All procedures were performed according to the ethical principles established under the Declaration of Helsinki. Eigthy single-rooted human permanent teeth without caries that were extracted for periodontal reasons were selected for this study. The teeth were cleaned of calculus, soft tissues and debris with hand instruments. The teeth were kept in $0.2 \%$ sodium azide solution until use.

Preparation of samples: Standard access preparations were prepared and the occlusal edges were flattened for reproducible reference point. The crown of each tooth was sectioned at the cemento--enamel junction using a diamond disk (DFS Diamon GmbH Landenstraße, Riebenburg, Germany), revolving at a conventional speed in order to simplify access to the root canal and establish a level surface to serve as a stable reference for all measurements (8). Coronal Preflaring was accomplished using \#4, \#3, and \#2 (Mani Inc., Japan) and pulp was extirpated with a barbed broach (Spirocolorinox, Dentsply).

Measurement of actual length: The actual lengths (AL) were determined using a \#10 file into the canal until the tip of the file was just visible at the apex using X 2.5 magnification measured with digital calipers (Mitutoyo Co., Japan).The actual working length was established by subtracting 0.5 $\mathrm{mm}$ from this measured length. The actual length readings were compared to the electronic working length readings. Apical instrumentation was completed to a \#20 (ISO) Flex- R file. The canals were then irrigated with $20 \mathrm{ml}$ of distilled water using 26 gauge irrigating syringe.

Working model for electronic length determination:Three plastic rectangular boxes, 15 $\times 3 \times 4 \mathrm{~cm}$ in dimension, were used for preparing this model. Ten teeth among the selected samples were glued to a plastic frame using modelling wax. Alginate was poured into the box, and the frame with the teeth was embedded into the alginate. In order to complete the electrical circuit of the apex locator, the labial clip was fixed to the edge of the plastic box and immersed in alginate. 
All the measurements were made within 2 hours of the model being prepared.

Electronic length (EL) measurements: This was carried out using four irrigants in the root canals;

- $0.9 \%$ Saline,(Claris life sciences Ltd,India)

- $3 \%$ Sodium hypochlorite (vishal Dentocare,India)

- $2 \%$ Chlorhexidine digluconate (Bombay dental surgicals Pvt Ltd,India)

- $17 \%$ EDTA liquid.(Bombay dental surgicals Pvt Ltd,India)

During study care was taken to ensure that all the circuits, batteries and the operating modes of both apex locators are fully functional. At first, canals were irrigated with 3\% Sodium Hypochlorite using 26 gauge beveled needle in $2 \mathrm{ml}$ syringe (Unolok, Hindustan Syringes and Medical Devices Ltd, India). The pulp chamber was then gently dried with an air syringe, and cotton pellets were used to dry the tooth surface and eliminate excess irrigating solution. A $15 \mathrm{~K}$-file (Mani, Tochigi, Japan) with the file clip of the apex locator was attached to the file and inserted inside the canal until the apex locator showed the "apex" reading. The file was slightly pulled out until the apex locator showed the "0.5 mm" reading. The silicone stop was adjusted, the file was removed and the distance between the base of silicone stop and file tip was measured using digital caliper (Mitutoyo Co., Japan) to the nearest $0.5 \mathrm{~mm}$; $0.5 \mathrm{~mm}$ was subtracted from this length and recorded as Actual Length (AL).Electronic Measurements were considered as valid if they were stable for at least 5 seconds. The recorded values were then tabulated.

The electronic length measurements of 40 teeth with Root ZX were recorded followed by 40 teeth using i-Root apex locator. The "apex" as indicated by both devices was chosen as the apical reference. Measurements were repeated three times and the average was computed. For each irrigant, 20 teeth were used, that is 10 teeth for each apex locator. Electronic length measurements were recorded using Root ZX followed by i- Root. For every irrigant, the order was reversed.

Each measurement was repeated three times and the average was calculated and computed. To prevent cross-contamination, each set of the two measurements was conducted in a fresh mixture of alginate. Between testing, teeth were irrigated with distilled water and dried with paper points. Results were subjected to statistical analysis.

\section{RESULTS}

Compilations of statistical analysis were done. Table 1 shows mean and standard deviation of distances between the apical constriction and the instrument tip with respect to actual length and electronic length measured using both electronic apex locators in the presence of four different irrigating solutions.

Table 1: Mean and Standard Deviation of distances between the apical constriction and the instrument tip with respect to Actual Length and Electronic Length (EL)

\begin{tabular}{|c|c|c|c|c|c|}
\hline & Media & $\mathrm{N}$ & Mean & $\begin{array}{l}\text { Standard } \\
\text { Deviation(SD) }\end{array}$ & $\begin{array}{l}\text { Standard } \\
\text { Error (SE) }\end{array}$ \\
\hline \multirow{4}{*}{ Actual length } & $\mathrm{NaOCl}$ & 20 & 19.75 & 2.38 & 0.53 \\
\hline & Chlorhexidine & 20 & 19.55 & 1.82 & 0.40 \\
\hline & EDTA & 20 & 19.4 & 1.18 & 0.26 \\
\hline & Saline & 20 & 20.52 & 1.60 & 0.35 \\
\hline EL of & $\mathrm{NaOCl}$ & 20 & 19.27 & 2.02 & 0.45 \\
\hline Root ZX & Chlorhexidine & 20 & 18.24 & 1.62 & 0.36 \\
\hline \multirow{5}{*}{$\begin{array}{l}\text { EL of } \\
\text { i-Root }\end{array}$} & EDTA & 20 & 18.78 & 1.31 & 0.29 \\
\hline & Saline & 20 & 20.76 & 1.53 & 0.34 \\
\hline & $\mathrm{NaOCl}$ & 20 & 19.14 & 2.19 & 0.49 \\
\hline & Chlorhexidine & 20 & 18.53 & 1.81 & 0.40 \\
\hline & EDTA & 20 & 18.62 & 1.17 & 0.26 \\
\hline
\end{tabular}


Table 2 indicates that the distances of the instrument tip from the apical constriction in both apex locators were not significant. However, the electronic measurements of both devices were shorter than the actual length in an average range between 0.51 $(0.15 \mathrm{SE})$ and 1.31 (0.17SE). Yet, only for
Chlorhexidine were the measurements slightly deviated from actual length, but it was not clinically significant. The presence of irrigating solution like saline, $\mathrm{NaOCl}$, chlorhexidine and EDTA in the canal did not have a significant effect on the accuracy of the Root ZX or i-Root $(\mathrm{P}<0.36)$.

Table 2: Distances of the Instrument tip from the Apical Constriction in both Apex Locators

\begin{tabular}{lcllc}
\hline & Root ZX ( SE ) & i- Root ( SE ) & t- value & p-value \\
\hline Saline & $0.51(0.15)$ & $0.27(0.11)$ & 1.86 & $0.17 \mathrm{NS}$ \\
NaOCl & $0.48(0.10)$ & $0.61(0.10)$ & 1.11 & $0.27 \mathrm{NS}$ \\
Chlorhexidine & $1.31(0.17)$ & $1.02(0.19)$ & 1.12 & $0.26 \mathrm{NS}$ \\
EDTA & $0.62(0.15)$ & $0.72(0.15)$ & 0.70 & $0.48 \mathrm{NS}$ \\
\hline
\end{tabular}

SE -Standard Error

However, as shown in Table 3, multiple comparision (Tukey HSD test) mesurments of both apex locators in relation to actual length (AL) indicated that measurement made with saline and its comparision with chlorhexidine (CHX) and EDTA showed a P value of $<0.05$.

Table 3: Post Hoc Test Tukey's Method

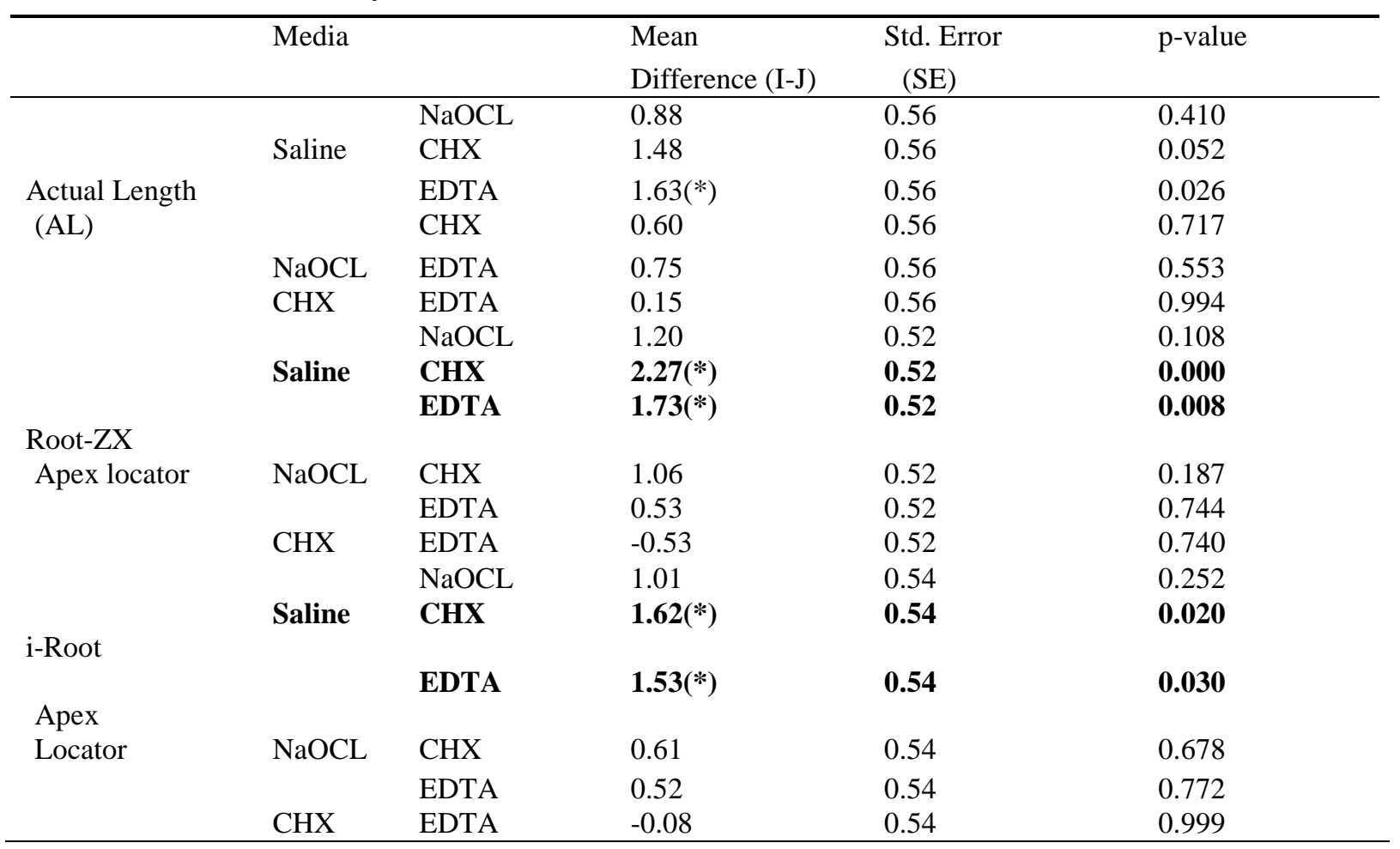

\section{DISCUSSION}

Apical constriction (minor diameter) may vary widely in shape; it is generally the narrowest portion of the root canal. It has the smallest diameter of blood supply. Therefore, creating the smallest wound site and this provides the best healing conditions (4).
Cemento-dentinal-junction (CDJ), the point where the pulp tissue changes into periodontal tissue, is the most ideal physiologic apical limit of working length. However, CDJ is highly irregular and can be upto $3 \mathrm{~mm}$ higher on one wall of the root compared with the opposite wall. Its relationship with minor constriction also varies.However, the CDJ and apical constriction do not always coincide, particularly in 
senile teeth where cementum deposition occurs continuously. This alters the position of the minor diameter(7). In this invitro study, two modern electronic apex locators, namely, Root ZX and iRoot were used to calculate the working length of the root canal.

This invitro study was designed to allow easy determination of the actual tooth length. The media used in mounting models need to have similar electrical resistance to periodontal tissue to allow for accurate data collection. Alginate has been described as the ideal embedding medium because its relatively firm consistency prevents intrusion of material into the apical foramen and resists force exerted by mechanical movement of the file, allowing the operator to accurately determine working length $(8$, 9).

The main disadvantage was in its limited working time because the alginate tended to desiccate unless kept in a moist environment. In this study, all measurements were made within 2 hours of the model being prepared in order to ensure that the alginate was kept sufficiently humid. The alginate model used in this study was accurate, easy to assemble and cost-effective.

To evaluate the accuracy of EALs, the \pm 0.5 $\mathrm{mm}$ range from AL was chosen. This is considered clinically acceptable and highly accurate (10).

Results of this study showed that the evaluated irrigant did not have any effect on the accuracy of the Root ZX and i -Root (11). This matches with results of studies carried out by different researchers (12) (13) and (14). In their study Root ZX and i-root apex locators were also tested along with other apex locators.

In their study Kang JA, Kim SK (12), evaluated accuracies of seven different apex locators under various conditions. EALs used were Apex Finder 7005, Apit, Bingo-1020, i-Root, ProPex, Root ZX and SmarPex. The measurements were taken when the canals were dry and saturated sequentially with $5.25 \% \mathrm{NaOCl}$, saline, $0.1 \%$ chlorhexidine and $15 \%$ EDTA. All these tested EALs were reliable in the presence of five root canal irrigants.

In another study by Sakkir $\mathrm{N}$ et al (13), it was revealed that no statistically significant differences were found amongst the five apex locators (Root ZX II, i-Root, Endo Master, Triauto ZX, and Elements apex locator) and the actual working length of the teeth evaluated. The large majority of EAL measurements were within the $\pm 0.5 \mathrm{~mm}$ of the actual length for all the five electronic apex locators. The average values of all groups indicated that; all the apex locators tested in this study can accurately determine the root canal length with the Root ZX II, Triauto ZX and iRoot apex locator readings being the closest to the actual root canal length.

Some scientists Niranjan A et al (14) did an invitro evaluation of the efficacy of five apex locators, namely, Root zx, Propex, Dentaport ZX, i-Root and Reypex 5. All these modern apex locators gave comparable results in comparison to actual working length. However, Root zx and i-Root were the most precise, followed by Dentaport zx, Raypex 5 and Propex.

In different research studies (15), Carvalho AL et al compared the accuracy of three electronic apex locators (EALs) - Elements Diagnostic $\AA$, Root ZX® and Apex DSP® - in the presence of different irrigating solutions $(0.9 \%$ saline solution and $1 \%$ sodium hypochlorite). Carvalho AL et al concluded that Elements Diagnostic ${ }^{\circledR}$ and Root ZX ®apex locators were able to locate the cementum-dentine junction more precisely than Apex DSP $®$. Moreover, the presence of irrigating solutions does not interfere with the performance of the EALs.

In another invitro study by Jain S (16) which compared the efficacy of Root ZX and Propex II EALs in the presence of $1 \% \mathrm{NaOCl}, 2 \% \mathrm{CHX}$ and LA Solution; $2 \%$ CHX matched more precisely with the actual canal length measurement.

In their invitro study Dinapadu S (17) checked Root ZX II accurate in the presence of $3 \% \mathrm{NaOCl}$ and $17 \%$ EDTA when measured with smaller and larger files. The results showed that Root Zx II was accurate in the presence of saline and 2\% CHX when larger files were used.

Another author Mull (18) designed invitro study to compare the accuracy of Root ZX and SybronEndo Mini, in the presence of $0.9 \%$ saline; $1 \%$ sodium hypochlorite $(\mathrm{NaOCl}) ; 2 \%$ chlorhexidine digluconate (CHX), and 17\% EDTA solution. Electronic measurements were shorter with $1 \%$ $\mathrm{NaOCl}$, whereas longer with $2 \% \mathrm{CHX}$ for both the devices. Sybron Mini was more accurate using 1\% $\mathrm{NaOCl}$ and 2\% CHX than Root ZX, when the measurements were compared.

In this study, with both apex locators, Saline and EDTA gave results close to the actual length. Thus, these irrigants can be considered as reliable solutions for electronic measurements. Large deviation occurred with the more conductive solutions such as $\mathrm{NaOCl}$ and Chlorhexidine digluconate. However, 2\% Chlorhexidine digluconate showed the largest deviation but 
clinically not significant. This is in agreement with the findings of another study (18).

Chlorhexidine digluconate is an antiseptic and has an affinity to hydroxyapatite(11). Thus, its use should be carefully monitored when indication of chlorhexidine is necessary for the treatment of apical areas of the root canal.

The use of irrigating solutions is an important aspect of endodontic treatment. The irrigants investigated were: $3 \% \mathrm{NaOCl}$, with tissue solvent and antibacterial activity; $2 \% \mathrm{CHX}$ with antibacterial activity even effective against Enterococcus faecalis; $17 \%$ EDTA, a chelator which facilitates canal preparation and removes the smear layer and $0.9 \%$ saline with only flushing action served as a control (11). Thus, $3 \% \mathrm{NaOCl}$ was used in this study because other invitro studies (19) indicate that the accuracy of EALs is not significantly influenced by different concentrations of $\mathrm{NaOCl}$.

Within its limitations of the present study, it can be concluded that both the Electronic Apex Locators that is Root ZX and i-Root can be used safely to determine working length in the presence of saline, EDTA, sodium hypochlorite and chlorhexidine. Previous studies have shown better results with Root ZX in the presence of irrigants used in their study, but our study showed that the same results can be achieved using root ZX or i-Root apex locator routinely in the practice of Endodontics for measuring the length of the root canal. The contents of the root canal did not influence the results of the measurements with both the electronic apex locators.

\section{REFERENCES}

1. Ingle J.I, Geoffrey S, Heithersay,Hartwell R.G. Endodontic diagnostic Procedures. Text book of Endodontics, 5th ed. Pliladelphia: Elsevier; 2003. p 228-307.

2. Sjo"gren U, Hagglund B, Sundqvist G, Wing K. Factors affecting the long-term results of endodontic treatment. J Endod 1990;16:498-504.

3. Kim E, Lee SJ. Electronic apex locator. Dent Clin North Am. 2004;48:35-54.

4. Nekoofar MH, Ghandi MM, Hayes SJ, Dummer PMH. The fundamental operating principles of electronic root canal length measurement devices. Int Endod J 2006;39:595-609.

5. Sunada New method for measuring the length of the root canal. Journal Dental Research.1962; 41:375-87.
6. Welk AR, Baumgartner JC, Marshall JG. An in vivo comparison of two frequency-based electronic apex locators. J Endod 2003;29:497-500.

7. Gordon MP, Chandler NP. Review: Electronic apex locators. Int Endod J 2004;37:425-37.

8. Kumar S, Chacko Y, Lakshminarayanan L. A simple model to demonstrate the working of electronic apex locators. $J$ Endod 2004;16:50-3.

9. Tinaz AC, Alacam T, Topuz O. A simple model to demonstrate the electronic apex locator. Int Endod $J$ 2002;35:940-5.

10. Guise GM, Goodell GG, Imamura GM. In vitro comparison of three electronic apex locators. $J$ Endod.2010;36:279-81.

11. Pommer O, Stamm O, Attin T. Influence of the canal contents on the electrical assisted determination of the length of root canals. $J$ Endod 2002;28:83-5.

12. Kang JA, Kim SK. Accuracies of seven different apex locators under various conditions. Oral Surg Oral Med Oral Pathol Oral Radiol Endod 2008;106(4):57-62.

13. Sakkir N, Asifulla M, Chandra V, Idris M, Razvi SF, GeetaI B. In vitro evaluation of the accuracy of five different electronic apex locators. Saudi Endod $J$ 2015;5:177-81.

14. Niranjan A,Vatkar Sucheta sathe, Vivek Hedge.In vitro evaluation of the efficacy of five apex locators. Endodontology 2010;24(4):36-42

15. Carvalho AL, Cacio MN, Maranhão de Moura AA,Márcia Martins Marques. Accuracy of three electronic apex locators in the presence of different irrigating solutions Braz. oral res2010;24(4):332.

16. Saru Jain and Ravi kapoor .Comparative evaluation of accuracy of two electronic apex locators in the presence of various irrigants: $\mathrm{An}$ in vitro study Contemp Clin Dent. 2012 ; 3 (Suppl 2): S140-S145.

17. Dinapadu S, Pasari S, Admala SR, Marukala NR, Gurram S, Peddi R. Accuracy of electronic apex locator in enlarged root canals with different root canal irrigants: an in vitro study. J Contemp Dent Pract. 2013;14(4):649-52.

18. Mull Paras J, Vinutha Manjunath, and MK Manjunath. Comparison of accuracy of two electronic apex locators in the presence of various irrigants: An in vitro study J Conserv Dent.2012; 15(2):178-182.

19. Tinaz AC, Sibel LS, Guliz G, Turkoz EG. The effects of sodium hypochlorite concentrations on accuracy of an apex locating device. J Endod. 2002;28:160-2. 